\title{
Punctures on Super Riemann Surfaces
}

\author{
Steven B. Giddings * \\ Department of Physics, University of California, Santa Barbara, CA 93106, USA
}

Received March 26, 1991

\begin{abstract}
An investigation of the geometry of punctured super Riemann surfaces and their moduli is pursued with particular emphasis on Ramond (or spin) punctures. A central role is played by the notion of a standard punctured neighborhood, which may be glued into a super Riemann surface to create a punctured surface. A geometrical explanation is given for the rule that two Ramond punctures only have one odd modulus. Explicit examples of spheres with Ramond and Neveu-Schwarz punctures are constructed and the connection between moduli of punctures and picture changing is elaborated. This description of punctures combined with the operator formalism should be useful both in treating fermionic or $(R, R)$ sector backgrounds for the superstring and in the unitarity proof for the superstring.
\end{abstract}

Much progress has been made on derivation of superstring amplitudes and elucidation of their properties in the Ramond-Neveu-Schwarz formulation. In this development a central role has been played by the supergeometry of super Riemann surfaces (SRSs) and their supermoduli spaces ${ }^{1}$. Goals in this development include proofs of the unitarity [3] and finiteness of the superstring (or heterotic string). A second set of goals is a better understanding of the general structure of string amplitudes, in particular with an eye towards understanding the basic variables in which string theory should be formulated and towards discovering the fundamental symmetries of string theory. Particularly promising in this direction has been the development of the operator formalism [4-6] which systematizes the derivation of string amplitudes, taking into account the many subtleties that have arisen, and may go beyond to point the way to a more fundamental formulation of string theory. The construction of the operator formalism for the superstring has in particular relied on aspects of the mathematics of SRSs.

Despite significant success in the treatment of superstring amplitudes, open issues remain. Some of these center on the proper inclusion of vertex operators,

\footnotetext{
* On leave from the Harvard Society of Fellows. Supported in part by D.O.E. Outstanding Junior Investigator Grant DE-AT03-76ER70023

${ }^{1}$ For reviews see $[1,2]$
} 
particularly in the Ramond sector, into amplitudes. Various motivations to seek a better understanding can be stated. One is the desire to understand the general perturbation of superconformal field theories; general perturbations may consist of fields either in the Neveu-Schwarz or Ramond sectors, and hence a systematic formalism for including both such backgrounds is desired. In particular, the problem of considering Ramond sector backgrounds (either spacetime fermions, or bosons in the $(R, R)$ sector of the type II string) is poorly understood at best. Application of the tools of SRS theory through the operator formalism can be expected to improve on this. In addition there is intrinsic mathematical interest: SRSs and their moduli spaces are interesting in their own right, and there are important questions regarding their global structures (e.g. splitness or projectedness of supermoduli space $\left.{ }^{2}, \ldots\right)$ that should be answered.

An improved understanding of the role of punctures on SRSs is therefore desirable. Two routes to this are better formulation of the general picture of punctures on SRSs and development of concrete and simple examples of punctured SRSs, e.g. the punctured spheres. These are the principal focus of this paper. In the next section the formulation of the moduli problem for punctured Riemann surfaces (RSs) or for SRSs with Neveu-Schwarz punctures is considered, using the notion of a standard punctured neighborhood, and followed by a discussion of special cases (spheres with NS punctures). The moduli problem for Ramond punctures is considered next, and some special cases (again spheres) are described. There follows a statement of the general features of the moduli problem and its connection with the space of meromorphic $3 / 2$ differentials, after which there is a brief discussion of the relationship between supermoduli and picture changing and a description of $\mathbf{R}$ punctures in the language of string scattering diagrams. In conclusion future directions are discussed.

\section{Standard Punctures on SRSs}

A first reasonable set of goals is to understand, in relatively concrete fashion, some fairly basic issues such as 1) how many supermoduli are needed to describe a punctured SRS 2) how (explicitly) do these supermoduli arise and 3) what are simple examples of supermoduli spaces for punctured SRSs. The answer to these questions is well known for ordinary RSs, reasonably well understood for NeveuSchwarz (N) punctures on SRSs, and somewhat murky for Ramond (R) punctures on SRSs. We consider them in that order.

The insertion of a puncture on a Riemann surface is quite simple and can be easily visualized by introducing the concept of a standard punctured neighborhood, or standard puncture for short. A standard puncture is a neighborhood $D_{0}$ of the origin in $\mathbb{C}^{1}$, with a complex structure and with the origin singled out as a preferred marked point. For an ordinary neighborhood with complex coordinate $z$ the allowed automorphisms of the neighborhood are all transformations $z \rightarrow f(z)$ with $f$ holomorphic on the neighborhood. Likewise, $z \rightarrow f(z)$ with holomorphic $f$ and

\footnotetext{
${ }^{2}$ Projectedness is of course now less physically interesting in light of arguments that it is irrelevant to the "ambiguities" of the superstring [7]
} 
$f(0)=0$ are the allowed automorphisms of the standard punctured neighborhood (or disk).

We can construct an unpunctured RS by gluing together ordinary neighborhoods with holomorphic transition functions; the set of all such transition functions, modulo these that can be removed by the holomorphic automorphisms of the neighborhoods, gives the $3 g-3$ (complex) dimensional moduli space according to the usual (Øech) approach. To get a punctured RS we substitute a standard puncture for one of the neighborhoods. This modifies the moduli problem because now there is one fewer transition function eliminated by the allowed automorphisms of the neighborhood onto itself (if $z$ is a coordinate in the punctured neighborhood, $z \rightarrow f(z)$ with $f(0) \neq 0$ is now forbidden), so there is one more modulus. This modulus is the position of the puncture.

$\mathrm{N}$ punctures on SRS can be similarly described. A standard $\mathrm{N}$ puncture is a neighborhood $D_{N}$ of the origin in $\mathbb{C}^{1 / 1}$, with inherited superconformal structure and with a fixed "point" $z=0=\theta$. To be more precise, the allowed automorphisms of $D_{N}$ onto itself are the superconformal transformations $(z, \theta) \rightarrow\left(z^{\prime}, \theta^{\prime}\right)$ with $z^{\prime}(0,0)=$ $0=\theta^{\prime}(0,0)$.

The above argument extends to show how the moduli problem for an SRS is modified by the insertion of a $\mathrm{N}$ puncture. First, a parametrized family of SRSs is constructed by gluing together superneighborhoods of $\mathbb{C}^{1 / 1}$ with patching functions superholomorphic on intersections. The supermoduli space is the space of all such patching functions, modulo those that can be eliminated by the superconformal automorphisms of the individual neighborhoods. This space is $(3 g-3 \mid 2 g-2)$ dimensional for general $g$. An $\mathrm{N}$ puncture may be inserted by replacing one of the neighborhoods by a standard $\mathrm{N}$ puncture. As before one less even transition function is eliminated by the automorphisms of the disk, since $z \rightarrow f(z, \theta)$ with $f(0,0) \neq 0$ is not allowed. Likewise one less odd transition function is eliminated because $\theta \rightarrow \phi(z, \theta)$ with $\phi(0,0) \neq 0$ is not allowed. The result is one even modulus and one odd modulus for the puncture, and these can be thought of as the even and odd "coordinates" of the puncture.

\section{Spheres with N Punctures}

Before considering surfaces with $\mathrm{R}$ punctures we review the construction of moduli spaces for spheres with $\mathrm{N}$ punctures. The extension of $S L(2, \mathbb{C})$ to the super-sphere is $\widehat{S L}(2, \mathbb{C})$, whose general transformation is of the form

$$
\begin{aligned}
& z \rightarrow\left[\frac{A z+B}{C z+D}+\theta \frac{\Gamma z+\Delta}{(C z+D)^{2}}\right] \frac{1}{1+\Delta \Gamma} \\
& \theta \rightarrow \frac{\theta}{C z+D}+\frac{\Gamma z+\Delta}{C z+D}
\end{aligned}
$$

with even parameters $A, B, C, D$ (such that $A D-B C=1$ ) and odd parameters $\Gamma, \Delta$. Thus the first example of a sphere with non-trivial moduli is that with three $\mathrm{N}$ punctures. The three even parameters of (2.1) can be used to set the even coordinates of the punctures to arbitrary values, but the two odd parameters fix only two of the odd coordinates, leaving one odd modulus. 
More explicitly, the NNN-punctured sphere can be constructed by taking $\mathbb{C}^{1 \mid 1}$, with coordinates $z, \theta$, removing three disks near $z=a, b, c$, and gluing in standard punctures. Taking $z_{a}, \theta_{a}$ as the local coordinates for the standard puncture to be glued in at $a$, the gluing map is

$$
z_{a}+\theta_{a} \alpha=z-a, \quad \theta_{a}+\alpha=\theta .
$$

We can then say that the puncture is "at" $z=a, \theta=\alpha$ (since these correspond to $\left.z_{a}=0=\theta_{a}\right)$, and likewise for punctures $(b, \beta)$ and $(c, \gamma)$. An ordinary $S L(2, \mathbb{C})$ transformation on $z, \theta$ may then be used to set $a, b$, and $c$ to arbitrary values (e.g. $0,1, \infty$ ), and then the remaining odd transformations in (2.1) may be used to set two of the odd coordinates to zero leaving a solitary odd modulus.

The existence of a single odd modulus on the NNN sphere suggests the existence of an invariant cross-ratio, in analogy to the cross ratio that arises by fixing three of the points $(a, b, c, d)$ on the ordinary four punctured sphere to $(0,1, \infty)$. In that case the coordinate

$$
x_{0}=\frac{(a-d)(b-c)}{(a-b)(d-c)}
$$

of the remaining point is the invariant cross ratio and may serve as an invariant parameter for the modulus. Likewise, if the three even coordinates on the NNN sphere are set to $(0,1, \infty)$ and the odd coordinates of the punctures at $1, \infty$ are set to zero, the odd coordinate of the puncture at $z=0$ gives an $\widehat{S L}(2, \mathbb{C})$ invariant cross ratio,

$$
\chi=\sqrt{\frac{1}{z_{a b} z_{c b} z_{a c}}}\left[\alpha z_{b c}+\beta z_{c a}+\gamma z_{a b}+\alpha \beta \gamma\right],
$$

where $z_{a b}=a-b-\alpha \beta$, etc. (This expression first appeared in [8].)

A slightly richer example is the sphere with four $\mathrm{N}$ punctures, which will have dimension $1 \mid 2$; this example was treated in $[9,1]$. Using $\widehat{S L}(2, \mathbb{C})$ we can take three of the points to have even coordinates $(0,1, \infty)$, and two of the points to have zero odd coordinates. So long as no two points coalesce, a good even coordinate is the generalization of $(2.3)$,

$$
x=\frac{z_{a d} z_{b c}}{z_{a b} z_{d c}}
$$

which is seen to be invariant by noting the transformation law

$$
z_{12} \rightarrow \frac{z_{12}}{\left[C z_{1}+D-\theta_{1}(\Gamma D-\Delta C)\right]\left[C z_{2}+D-\theta_{2}(\Gamma D-\Delta C)\right]}
$$

under (2.1). Likewise good odd coordinates will be two of the odd cross ratios. However, when points collide caution is required. The proper way to proceed is to carefully study the degenerating surface near the compactification divisor.

Fix the coordinates of three of the points to $(a, \alpha),(b, \beta)$, and $(c, 0)$, and let the coordinates of the fourth roaming point be $(w, 0)$. As $w \rightarrow a$ we can construct a degenerating surface by sewing together two spheres, as in Fig. 1, using the plumbing 


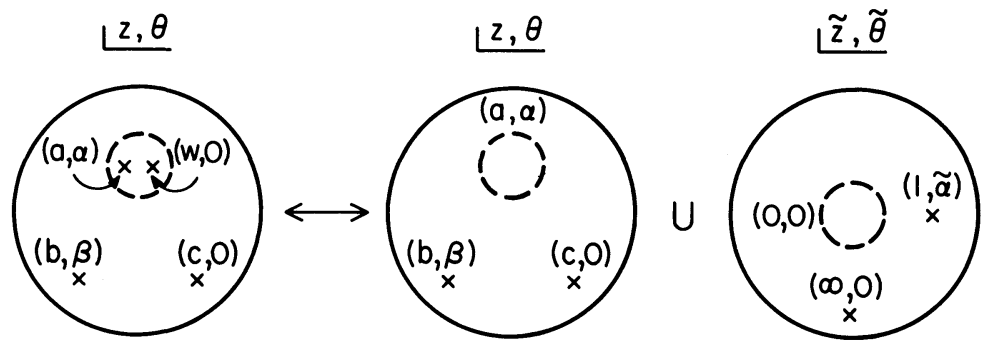

Fig. 1. The degeneration of an SRS when two Neveu-Schwarz punctures collide is described in terms of the pictured plumbing construction

fixture transformation [10]

$$
\tilde{z}=\frac{w-a}{z-a-\theta \alpha}, \quad \tilde{\theta}=i(\theta-\alpha) \frac{\sqrt{w-a}}{z-a} .
$$

The roaming point is then mapped to $(1, \tilde{\alpha})$ with $\tilde{\alpha}=\alpha / \sqrt{a-w}$. Thus good (holomorphic) coordinates near the divisor are $t_{a}=\sqrt{a-w}, \tilde{\alpha}=\alpha / t_{a}$, and $\beta$. This indicates that the moduli space should be thought of as a branched cover of the sphere. ${ }^{3}$ Similarly when $w$ and $b$ collide, the good coordinates are $t_{b}=\sqrt{b-w}$, $\tilde{\beta}=\beta / t_{b}$, and $\alpha$.

The degeneration as $w \rightarrow c$ is more complicated. As it stands, if we consider a plumbing fixture like (2.7) then in the limit we have two thrice-punctured spheres, one with two non-zero odd coordinates for the punctures and the other with all odd coordinates zero. This does not correspond to the stable compactification. Instead we must move one of the odd moduli onto $c$ before pinching the surface. The transformation

$$
\theta^{\prime}=\theta-\beta \frac{z-w}{b-w}, \quad z^{\prime}=z-\theta \beta \frac{z-w}{b-w}
$$

moves the punctures to $\left(a^{\prime}, \alpha^{\prime}\right),(b, 0),\left(c, \gamma^{\prime}\right),(w, 0)$ with

$$
\alpha^{\prime}=\alpha-\beta \frac{a-w}{b-w}, \quad \gamma^{\prime}=-\beta \frac{c-w}{b-w} .
$$

In these new coordinates the plumbing fixture for $w \rightarrow c$ is

$$
\tilde{z}=\frac{-t_{c}^{2}}{z^{\prime}-c-\theta^{\prime} \gamma^{\prime}}, \quad \tilde{\theta}=t_{c} \frac{\theta^{\prime}-\gamma^{\prime}}{z^{\prime}-c}
$$

with $t_{c}=\sqrt{c-w}$. Analytic coordinates for supermoduli space near the pinch are $t_{c}$ and the new odd coordinates of the punctures,

$$
\tilde{\gamma}=\frac{\beta t_{c}}{c-b}, \quad \tilde{\alpha}=\alpha-\beta \frac{a-c}{b-c} .
$$

\footnotetext{
${ }^{3}$ Ramification at the compactification divisor was pointed out by Deligne [11]
} 
From these results we learn that two of the three odd cross ratios

$$
\begin{aligned}
\chi_{a b w} & =\sqrt{\frac{1}{(a-b)(w-b)(a-w)}}[\alpha(b-w)+\beta(w-a)], \\
\chi_{b c w} & =\sqrt{\frac{w-c}{(b-c)(w-b)} \beta} \\
\chi_{a c w} & =\sqrt{\frac{w-c}{(c-a)(w-a)}} \alpha
\end{aligned}
$$

are nondegenerate analytic odd supermoduli at each degeneration $w \rightarrow a, b, c$. We can also use the even cross ratio,

$$
x=\frac{(a-b-\alpha \beta)(w-c)}{(a-w)(b-c)}
$$

to define analytic even coordinates in patches; good local coordinates are $\sqrt{x}$ near $w=c, 1 / \sqrt{x}$ near $w=a$, and $\sqrt{x-1}$ near $w=b .^{4}$

Examples of supermoduli spaces for surfaces with more $\mathbf{N}$ punctures can be analogously constructed, and can be parametrized using the invariant cross ratios, but this will not be pursued here.

\section{Standard R Punctures on SRSs}

The case of $\mathbf{R}$ punctures is slightly more complicated than that of $\mathbf{N}$ punctures. There is no such thing as inserting one puncture (since $\theta \rightarrow-\theta$ as we circle a puncture), so we might imagine using standard bi-punctured neighborhoods. However, a natural conjecture is that the automorphisms of these neighborhoods are superconformal transformations that leave fixed both the odd and even coordinates of the two punctures, and from this we might conclude that there is one even and one odd modulus per puncture as in the $\mathbf{N}$ case. This is wrong, as one can see for example by deriving the dimension of the space of holomorphic $3 / 2$ differentials: it is well known that there is only one odd modulus per pair of $\mathbf{R}$ punctures.

One common perception in the literature is that, because of the requirement that $\theta \rightarrow-\theta$ around a puncture, fewer transition functions are allowed when gluing the bipunctured neighborhood into the surface. This is not entirely correct, however, as one can see by noting that the properties of the gluing functions are local and do not depend on the global $\theta \rightarrow-\theta$. Put another way, if the two $\mathrm{R}$ punctures are very close to each other they are nearly indistinguishable from a $\mathbf{N}$ puncture, and in this case we would not say there is any restriction on the allowed patching data. Hence it's not clear how an argument of this type can reduce the number of moduli by one.

${ }^{4}$ As discussed in [1], near $w=b$ this choice of coordinate is not a split function of the natural plumbing fixture coordinate $t_{b}$, but that will not concern us here 
The simplest case where this moduli counting appears is the sphere with two $R$ punctures and one $\mathbf{N}$. If there were one odd modulus per puncture then this surface would have one odd modulus as in the case NNN; instead it has zero.

The reason for this can be understood by considering the super-torus, with odd spin structure, which we know reduces to an $\mathrm{R}$ punctured sphere upon factorization. We can describe this torus as the quotient of $\mathbb{C}^{1 / 1}$, with coordinates $(w, \eta)$, by the group of transformations $(w, \eta) \rightarrow(w+1, \eta),(w, \eta) \rightarrow(w+\tau+\eta \delta, \eta+\delta)$. If one $\mathbf{N}$ puncture is placed on this torus, at first sight there would appear to be two odd moduli, $\delta$ and the odd coordinate of the $\mathrm{N}$ puncture. This is not correct due to the existence of a conformal Killing spinor on the torus. The Killing spinor generates the transformation

$$
\eta \rightarrow \eta+\varepsilon, \quad w \rightarrow w+\eta \varepsilon
$$

which is compatible with the torus transition functions. ${ }^{5}$ This transformation can be used to shift away the odd coordinate of the $\mathrm{N}$ puncture. The persists when we pass to the limit in which the torus degenerates into the RRN sphere. (The remaining odd modulus $\delta$ belongs to the plumbing fixture [10], and therefore disappears in the degenerate limit.) In terms of the geometry of the sphere, one can explain the absence of a modulus by noting that the transformation (3.1) takes the form

$$
\theta \rightarrow \theta+\varepsilon \sqrt{\left(z-z_{1}\right)\left(z-z_{2}\right)}, \quad z \rightarrow z+\theta \varepsilon \sqrt{\left(z-z_{1}\right)\left(z-z_{2}\right)}
$$

where $z_{1}, z_{2}$ are the even coordinates of the $\mathbf{R}$ punctures, in the degeneration limit. If we think of the construction of the RRN sphere in terms of gluing a standard $\mathrm{N}$ puncture onto the RR sphere, then the odd coordinate of the $\mathrm{N}$ puncture can be eliminated by (3.2).

This motivates a definition of a standard $R$ puncture. We may take a disk surrounding the origin in the plane $\mathbb{C}^{1 / 1}$ ramified twice at the origin, with the identification

$$
z \rightarrow e^{2 \pi i} z, \quad \theta \rightarrow-\theta .
$$

The allowed automorphisms of this standard R-punctured disk $D_{R}$ are the superconformal transformations that respect this ramified structure, and vanish at the puncture. Thus in particular they include the transformation

$$
\theta \rightarrow \theta+\varepsilon \sqrt{z}, \quad z \rightarrow z+\theta \varepsilon \sqrt{z}
$$

with arbitrary constant parameter $\varepsilon .^{6}$

In the operator formalism (3.4) is the transformation generated by $G_{0}$. As discussed in $[6,1]$ an alternate description of $R$ punctures is in terms of the nondegenerating (but also non-superconformal) coordinates $(z, \eta)$, with $\eta=\theta / \sqrt{z}$. (Note that the use of $\eta$ both here and above is no notational accident.) In these coordinates

\footnotetext{
${ }^{5}$ Note, however, the interesting fact that this transformation also generates a nilpotent shift in the even modulus if $\delta \neq 0$, and thus the symmetry should properly be thought of as acting non-trivially on the parametrized family of odd tori

${ }^{6}$ The relevance of such supersymmetry transformations to $\mathbf{R}$ punctures was previously realized by Knizhnik [12]
} 
(3.4) becomes

$$
\eta \rightarrow \eta+\varepsilon, \quad z \rightarrow z(1+\eta \varepsilon) .
$$

The standard $R$ punctures may be glued (in pairs) into an arbitrary SRS. If we think of gluing in a bi-punctured neighborhood, as above, the additional modulus that we might have expected is eliminated by an the "extra" automorphism (3.2) of the disk with two $\mathrm{R}$ punctures. It is this extra automorphism that accounts for the fact that only one odd modulus is generated when two $R$ punctures are glued into an arbitrary SRS.

\section{Spheres with $\mathbf{R}$ Punctures}

Simple examples of these facts may again be demonstrated on the sphere. The next non-trivial case is that with two $\mathbf{N}$ punctures and two $\mathrm{R}$ punctures; the expected dimension of supermoduli space is $1 \mid 1$. This can be seen explicitly by gluing the punctures into the sphere coordinatized by $(z, \theta)$, as in Fig. 2 . Let the $\mathrm{N}$ punctures be glued in with coordinates $(a, \alpha)$ and $(b, \beta)$, and the $\mathrm{R}$ punctures have even coordinates $c, d$. Therefore $\theta \rightarrow-\theta$ as $z$ circles $c$ or $d$. The gluing maps for the $\mathrm{N}$ punctures are

$$
\begin{aligned}
& \theta_{a}+\alpha=\frac{\theta}{\sqrt{(z-c)(z-d)}}, \quad z_{a}+\theta_{a} \alpha=\frac{1}{c-d} \ln \left[\frac{z-c}{z-d} \frac{a-d}{a-c}\right], \\
& \theta_{b}+\beta=\frac{\theta}{\sqrt{(z-c)(z-d)}}, \quad z_{b}+\theta_{b} \beta=\frac{1}{c-d} \ln \left[\frac{z-c}{z-d} \frac{b-d}{b-c}\right],
\end{aligned}
$$

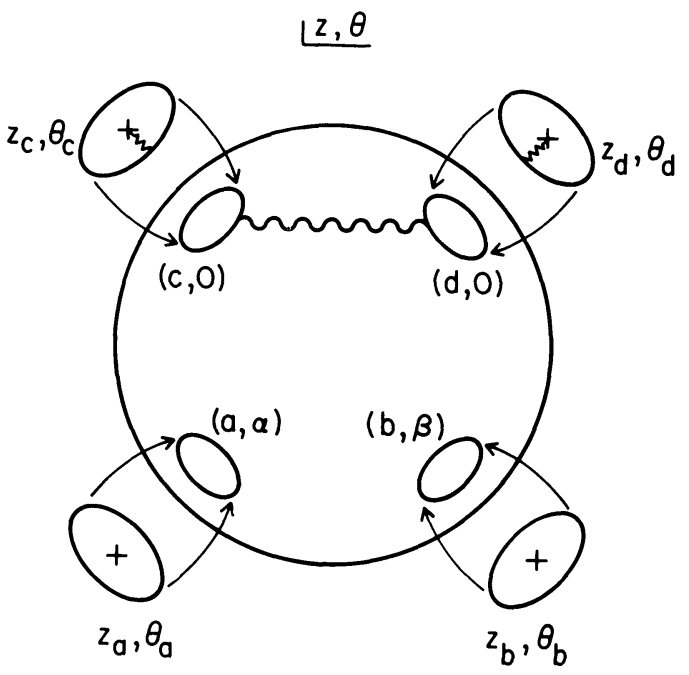

Fig. 2. The sphere with Ramond punctures at $c$ and $d$ and Neveu-Schwarz punctures at $a$ and $b$ is constructed by gluing standard punctures into the branched cover of the standard sphere 
and those for the $\mathrm{R}$ punctures are

$$
\begin{aligned}
& \theta_{c}=\frac{\theta}{\sqrt{z-d}}, \quad z_{c}=\ln \left[\frac{z-d}{c-d}\right], \\
& \theta_{d}=\frac{\theta}{\sqrt{z-c}}, \quad z_{d}=\ln \left[\frac{z-c}{d-c} \cdot\right],
\end{aligned}
$$

where $\left(z_{a}, \theta_{a}\right)$, etc., are the coordinates for the standard punctured neighborhoods.

At first glance one would expect the two odd coordinates to serve as odd moduli, but a transformation of $z, \theta$ of the form (3.2) removes one of them and can be absorbed into the $\mathrm{R}$ punctures. One can alternately shift the odd modulus from the transition functions gluing in the $\mathbf{N}$ punctures to those gluing in the $\mathbf{R}$ punctures; this can for example be accomplished by taking

$$
\theta=\theta^{\prime}+\sqrt{\frac{z-d}{z-c}}\left[\alpha \frac{z-b}{a-b}(a-c)+\beta \frac{z-a}{b-a}(b-c)\right]
$$

and the corresponding equation for $z^{\prime}$. This eliminates $\alpha$ and $\beta$, but cannot be eliminated by an allowed automorphism of the $R$ punctures because of the $1 / \sqrt{z-c}$, so can be thought of as moving the odd modulus to the $\mathrm{R}$ puncture at $c$. More generally, the $R$ punctures could have been glued in with

$$
\begin{aligned}
& \theta_{c}+\frac{\gamma}{\sqrt{z-c}}=\frac{\theta}{\sqrt{z-d}}, \quad z_{c}+\frac{\theta_{c} \gamma}{\sqrt{z-c}}=\ln \left[\frac{z-d}{c-d}\right], \\
& \theta_{d}+\frac{\delta}{\sqrt{z-d}}=\frac{\theta}{\sqrt{z-c}}, \quad z_{d}+\frac{\theta_{d} \delta}{\sqrt{z-d}}=\ln \left[\frac{z-c}{d-c}\right] .
\end{aligned}
$$

A transformation

$$
\theta \rightarrow \theta+\varepsilon \sqrt{(z-c)(z-d)}+\phi \sqrt{\frac{z-c}{z-d}}+\imath \sqrt{\frac{z-d}{z-c}}
$$

with odd parameters $\varepsilon, \phi, l$, can then be used to eliminate three of the four coordinates $\alpha, \beta, \gamma, \delta$.

It is also instructive to study the global structure of this supermoduli space, that is, to investigate its form near the compactification divisor. A shift (3.2) so that $\beta=0$ simplifies the problem; let $w \equiv b$ be the even coordinate of moduli space, and fix the position of the other three punctures. As $w \rightarrow a$ we encounter the same behaviour as before; the surface pinches into two spheres with RRN and NNN punctures. The good local coordinates for supermoduli are $t_{a}=\sqrt{a-w}$ and $\tilde{\alpha}=\alpha / t_{a}$.

The limit $w \rightarrow c, d$ produces the new phenomenon of a spin pinch. A naïve analysis would imply that the surface splits into two RRN spheres but that the odd coordinate of the $\mathrm{N}$ puncture on one of them is non-zero. However, this coordinate is removable as the RRN sphere has no moduli. An alternate viewpoint is that the odd modulus disappears into the spin-plumbing fixture as in [10]. To see this as $w \rightarrow c$ it is convenient to first map the two $\mathrm{R}$ punctures to 0 and $\infty$ and 
the fixed $\mathrm{N}$ puncture to 1 using the $S L(2, \mathbb{C})$ transformation

$$
\check{z}=\frac{(z-c)(a-d)}{(z-d)(a-c)}, \quad \check{\theta}=\sqrt{\frac{(a-d)(c-d)}{a-c}} \frac{1}{z-d} \theta ;
$$

the other $\mathrm{N}$ puncture is then at

$$
\check{w}_{c}=\frac{(w-c)(a-d)}{(w-d)(a-c)} .
$$

The plumbing transformation is

$$
\tilde{z}=\frac{\check{w}_{c}}{\check{z}}, \quad \tilde{\theta}=\frac{\sqrt{-\check{w}_{c}}}{\check{z}} \check{\theta}
$$

but does not give the desired compactification unless we shift the odd coordinate of the fixed $\mathrm{N}$ puncture to zero using

$$
\check{\theta}=\hat{\theta}+\alpha \sqrt{(c-d) \check{z}}, \quad \check{z}=\hat{z}+\check{\theta} \alpha \sqrt{(c-d) \check{z}} .
$$

Combining (4.8) and (4.9) gives

$$
\sqrt{-\tilde{z}}\left(\sqrt{\hat{z}}+\frac{1}{2} \alpha \hat{\theta} \sqrt{c-d}\right)=\check{t}_{c} ; \quad \tilde{\theta} \sqrt{\hat{z}}=\sqrt{-\tilde{z}} \hat{\theta}+\check{t}_{c} \alpha \sqrt{c-d}
$$

with $\check{t}_{c}=\sqrt{-\check{w}_{c}}$. This is the same as the spin plumbing transformation of [10], with $\tau_{c}=\alpha \sqrt{c-d}$ the analytic odd coordinate of the plumbing fixture. Thus one concludes that $t_{c}=\sqrt{c-w}$ and $\alpha$ are good coordinates near the compactification divisor at $c$; likewise near $d$ good coordinates are $t_{d}, \alpha$. The fact that $\alpha / t_{a}$ is the good coordinate when the two $\mathbf{N}$ punctures collide implies that the odd modulus transforms nontrivially over the body of supermoduli space, which is the sphere branched at $a, c, d$.

As in the case of the NNN sphere we might hope to define natural cross ratios for punctures glued in at arbitrary locations as in (4.1), (4.2). ${ }^{7}$ This can be done by moving all of the moduli onto the puncture originally at $a, \alpha$. The transformation

$$
\theta=\theta^{\prime}+\beta \sqrt{(z-c)(z-d)}, \quad z=z^{\prime}+\theta \beta \sqrt{(z-c)(z-b)}
$$

eliminates the odd coordinate at $b$, and then

$$
\hat{z}=\frac{\left(z^{\prime}-c\right)(b-d)}{\left(z^{\prime}-d\right)(b-c)}, \quad \hat{\theta}=\sqrt{\frac{(b-d)(c-d)}{b-c}} \frac{\theta^{\prime}}{z-d}
$$

moves $c, b, d$ to $(0,1, \infty)$. The resulting coordinates of the puncture formerly at $(a, \alpha)$ are

$$
\hat{a}=\frac{(a-c)(b-d)}{(a-d)(b-c)}[1+\alpha \beta(d-c)], \quad \hat{\alpha}=\sqrt{c-d}(\alpha-\beta) .
$$

\footnotetext{
${ }^{7}$ It proves difficult to define a cross ratio if the odd coordinates of the $\mathrm{R}$ punctures are not zero to begin with, since in that case the even coordinate of the puncture is ambiguous by a nilpotent piece due to (3.4)
} 
These are the natural even and odd cross ratios for the sphere with two $\mathrm{N}$ and two $\mathrm{R}$ punctures. They are easily seen to be invariant under both $S L(2, \mathbb{C})$ and (3.2) (i.e. under transformations generated by $G_{0}$.)

Another example is the sphere with four or six $R$ punctures, with $1 \mid 0$ and $3 \mid 1$ moduli respectively. In the case of RRRR suppose that the $R$ punctures are initially glued into the sphere, which has coordinates $(z, \theta)$, at even locations $a, b, c, d$. The gluing transformations are (generalizing (4.4))

$$
\begin{aligned}
\frac{\theta_{a}}{\sqrt{z-a}}+\frac{\alpha}{z-a} & =\frac{\theta_{b}}{\sqrt{z-b}}+\frac{\beta}{z-b}=\frac{\theta_{c}}{\sqrt{z-c}}+\frac{\gamma}{z-c} \\
& =\frac{\theta_{d}}{\sqrt{z-d}}+\frac{\delta}{z-d}=\frac{\theta}{\sqrt{(z-a)(z-b)(z-c)(z-d)}},
\end{aligned}
$$

with corresponding transformations for the even coordinates. Allowed transformations of the coordinates $z, \theta$ are

$$
\theta \rightarrow \theta+\zeta(z) \sqrt{(z-a)(z-b)(z-c)(z-d)}
$$

with $\zeta \sim 1 / z$ necessary for the transformation to be holomorphic at infinity. Thus we may use a $\zeta$ of the form

$$
\zeta=\frac{\tilde{\alpha}}{z-a}+\frac{\tilde{\beta}}{z-b}+\frac{\tilde{\gamma}}{z-c}+\frac{\tilde{\delta}}{z-d}
$$

to remove all of the odd coordinates. This confirms the absence of odd moduli; ordinary $S L(2, \mathbb{C})$ implies that there is one even modulus.

In the case of RRRRRR, modify the transformation (4.14) to include punctures with coordinates $(e, \varepsilon)$ and $(f, \phi)$. Now the allowed transformations of the $z, \theta$ plane are

$$
\theta \rightarrow \theta+\zeta(z) \sqrt{(z-a)(z-b)(z-c)(z-d)(z-e)(z-f)}
$$

where $\zeta \sim 1 / z^{2}$ for holomorphicity at infinity. Therefore

$$
\zeta=\frac{\tilde{\alpha}}{z-a}+\frac{\tilde{\beta}}{z-b}+\frac{\tilde{\gamma}}{z-c}+\frac{\tilde{\delta}}{z-d}+\frac{\tilde{\varepsilon}}{z-e}+\frac{\tilde{\phi}}{z-f},
$$

with the additional constraint $\tilde{\alpha}+\tilde{\beta}+\tilde{\gamma}+\tilde{\delta}+\tilde{\varepsilon}+\tilde{\phi}=0$, may be used to eliminate all but one of the odd coordinates, leaving one odd modulus. Ordinary $S L(2, \mathbb{C})$ reduces the even moduli to three.

\section{Generalities of Moduli for R Punctures}

The above discussion extends to completely general SRS. One may begin with an arbitrary SRS, pass to its double cover ramified over two points, and glue in a pair of standard $\mathbf{R}$ punctures at those two points. The moduli counting of two even and one odd moduli per pair continues to the general case; explicit construction of the resulting punctured SRS is simply a generalization of the above.

We may also relate this moduli counting to the properties of the space of holomorphic 3/2-differentials. Recall that on ordinary RSs deformations of transition 
functions may be represented by Beltrami differentials which in turn are dual to the quadratic differentials. In that case the Beltrami differentials are of the form $m=\bar{\partial} v$, where $\delta z_{(\alpha)}=v_{(\alpha)}^{z}$ is the variation of the coordinate $z_{(\alpha)}$ on patch $U_{\alpha}$ of the surface. The dual pairing between the quadratic differentials $\phi$ and the Beltrami differentials thus becomes

$$
\langle m, \phi\rangle=\oint_{C} d z \Delta v^{z} \phi_{z z}
$$

where $\Delta v$ is the discontinuity in $v$ along contours $C$ corresponding to patch overlaps. In the case where the modulus is the position of a puncture, $C$ is a circle surrounding the puncture, and $\Delta v^{z} \propto 1$, so meromorphic quadratic differentials with simple poles at the punctures are dual to the Beltrami differentials that move the punctures. The dimension of the space of such quadratic differentials indeed coincides with the dimension of the moduli space of the punctured surface on which the differentials live.

This logic continues to SRS. Here the superbeltrami differentials arise from the odd parts of the transition functions. In the case of punctures these take forms like (2.2) or (4.4), and the natural pairing with the $3 / 2$ differentials $\psi$ is

$$
\langle\mu, \psi\rangle=\oint_{C} d z \Delta v^{\theta} \psi_{z \theta}
$$

where $\mu=\bar{\partial} v$ and $\delta \theta_{(\alpha)}=v_{(\alpha)}^{\theta}$. Again $C$ is a contour surrounding the puncture $z_{0}$. For an $\mathrm{N}$ puncture, $\Delta v^{\theta} \propto 1$ and the corresponding $3 / 2$ differential has a simple pole at the puncture. For an $\mathrm{R}$ puncture, $\Delta v^{\theta} \propto 1 / \sqrt{z-z_{0}}$ as in (4.4) so $3 / 2$ differentials that behave like $1 / \sqrt{z-z_{0}}$ at the punctures correspond to changes of the odd coordinates at the puncture [12]. The space of meromorphic (and multivalued) $3 / 2$ differentials with these singularities at the punctures will have dimension equal to the odd dimension of supermoduli space.

Examples of this are the spheres considered in the previous section. For a $3 / 2$ differential to be holomorphic at $z=\infty$ it must behave as $1 / z^{3}$ there. Thus the first case of a non-zero holomorphic $3 / 2$ differential is the NNN sphere, where $\psi=d z^{3 / 2} /(z-a)(z-b)(z-c)$ corresponds to the single odd modulus. Likewise $\psi=d z^{3 / 2} /(z-a)(z-b) \sqrt{(z-c)(z-d)}$ corresponds to the single odd modulus for RRNN, and so on.

\section{Connection to Picture Changing}

The above treatment of the odd moduli gives a nice interpretation ${ }^{8}$ of the different pictures of vertex operators in the superstring $[13,12]$; the operators whose odd coordinates we take to be odd moduli are in different pictures from those whose odd coordinates are fixed.

To see this let $t^{i}$ and $\tau^{\alpha}$ be the even and odd moduli, $X$ the matter superfields, and $C=c+\gamma \theta, B=\beta+\theta b$ the ghosts. Recall that a superstring amplitude with $n$

${ }^{8}$ See also [6] 
$\mathrm{N}$ punctures and $r \mathrm{R}$ punctures takes the form

$$
\begin{aligned}
\mathscr{A}= & \int \mathscr{D} X \mathscr{D} B \mathscr{D C}\left|\prod_{i=1}^{3 g-3+n+r} d t^{i}\left\langle m_{i}, b\right\rangle\right|^{2}\left|\prod_{\alpha=1}^{2 g-2+n+r / 2} d \tau^{\alpha} \delta\left(\left\langle\mu_{\alpha}, \beta\right\rangle\right)\right|^{2} \\
& \cdot e^{-S[X, B, C]}\left(\mathcal{O}_{1} \cdots \mathcal{O}_{N}\right),
\end{aligned}
$$

where $\mathcal{O}_{I}$ are the vertex operators corresponding to external states. In this expression

$$
S[X, B, C]=S[X, B, C]_{\tau=0}+\sum_{\alpha} \tau^{\alpha}\left\langle\mu_{\alpha}, T_{F}\right\rangle,
$$

where $T_{F}$ is the supercurrent, $T_{F}=\gamma b / 2-3 \partial c \beta / 2-c \partial \beta$. Therefore after integrating over the odd moduli there is a factor of $\left\langle\mu_{\alpha}, T_{F}\right\rangle \delta\left(\left\langle\mu_{\alpha}, \beta\right\rangle\right)$ left for each odd modulus, as described in [14]. In particular, the odd moduli for the punctures may be taken to be the odd coordinates of the punctures, as above, and so (5.2) gives insertions of

$$
\delta\left(\oint \frac{d z}{\sqrt{z-z_{\alpha}}} \beta\right) \oint \frac{d z}{\sqrt{z-z_{\alpha}}} T_{F}
$$

for each $\mathbf{R}$ puncture with variable odd coordinate and

$$
\delta(\oint d z \beta) \oint d z T_{F}
$$

for each unfixed $\mathrm{N}$ puncture. The "natural" pictures for vertex operators before including these factors are $(-1)$ for $N$ states and $(-1 / 2)$ for $R$ states $[14,6]$. The extra insertions (6.3), (6.4) are precisely the picture changing operators of $[13,12]$ and convert $(r-4) / 2$ of the $R$ punctures to the (1/2) picture, and $n-2$ of the $N$ punctures to the $(0)$ picture.

One can also extend this discussion to higher genus. In [14] a delta function basis for the gravitini $\mu_{\alpha}$ was taken, but other alternatives are to either choose gravitini corresponding to higher order singularities at the punctures or to define the gravitini using $v^{\theta}$ 's that are discontinuous across other path boundaries on the surface. The former has the effect of putting some of the vertex operators in higher pictures, and the latter introduces contour integrals of picture changing operators similar to those arising in the bosonic string [15]. These prescriptions may be more practical for computation.

The results also provide a geometrical interpretation of the rearrangement lemma [16], which states that correlation functions for $\mathrm{R}$ states on the sphere obey

$$
\left\langle\cdots V_{1 / 2}(c) \cdots V_{-1 / 2}(d) \cdots\right\rangle=\left\langle\cdots V_{-1 / 2}(c) \cdots V_{1 / 2}(d) \cdots\right\rangle,
$$

where $V_{1 / 2}, V_{-1 / 2}$ are $\mathrm{R}$ vertex operators in the $( \pm 1 / 2)$ pictures. Indeed, the left side of (6.5) follows directly from the above discussion if one of the odd moduli is taken to reside at $c ;(6.5)$ is derived by moving this odd modulus from $c$ to $d$.

To accomplish this, take $c$ and $d$ to both lie in a single patch with coordinates $z, \theta$; the punctures are at first glued into this patch according to (4.4) with $\delta=0$. A shift in $\theta$ can then be used to set $\gamma=0$. In the general supermoduli problem for the sphere this transformation should leave the odd moduli of two other points fixed; these points correspond to other punctures whose odd coordinates have been set to zero by $\widehat{S L}(2, \mathbb{C})$. Let these points have even coordinates $z=a, b$. The 
desired transformation is

$$
\theta=\theta^{\prime}+\gamma \sqrt{\frac{z-d}{z-c}}\left(1-\frac{(z-c)(z-a)(b-d)}{(b-c)(b-a)(z-d)}-\frac{(z-c)(z-b)(a-d)}{(a-c)(a-b)(z-d)}\right)
$$

From (4.4), (6.6) we find the identification at $d$,

$$
\theta_{d}=\frac{\theta^{\prime}}{\sqrt{z-c}}+\gamma \frac{\sqrt{z-d}}{z-c}-\frac{\gamma}{\sqrt{z-d}}\left(\frac{(z-a)(b-d)}{(b-c)(b-a)}+\frac{(z-b)(a-d)}{(a-c)(a-b)}\right) .
$$

After an allowed redefinition of $\theta_{d}$ this reduces to

$$
\theta_{d}+\frac{\delta^{\prime}}{\sqrt{z-d}}=\frac{\theta^{\prime}}{\sqrt{z-c}}
$$

with $\delta^{\prime}$ given in terms of $\gamma, a, b, c, d$. Taking $\delta^{\prime}$ to be a new odd coordinate for moduli space, integration then pulls down a picture changing operator producing the operator $V_{1 / 2}(d)$. Changes in the odd coordinates of the other punctures are also induced by (6.6) but their integrated contributions remain unaltered since these changes are just shifts proportional to $\delta^{\prime}$.

Likewise this argument would appear to extend to higher genus. Again, it is possible to take any two $\mathrm{R}$ punctures to lie in a single patch with coordinates $z, \theta$, and to move the odd modulus from $c$ to $d$ using a transformation

$$
\theta=\theta^{\prime}+\gamma \sqrt{\frac{z-d}{z-c}}+\delta(z) \sqrt{\frac{z-c}{z-d}}+\varepsilon(z) \sqrt{(z-d)(z-c)} .
$$

The apparent arbitrariness of $\delta(z), \varepsilon(z)$ is fixed, in analogy to above, by the condition that change in the transition functions induced by (6.9) be removable (with only shifts in the other odd moduli); this determines the odd modulus $\delta(d) \sqrt{d-c}$ of the $\mathrm{R}$ puncture at $d$ in terms of $\gamma$.

It also seems likely that the formalism of this paper can provide a more geometrical description of spacetime supersymmetry. Indeed, the spacetime supersymmetry algebra arises from the operator product of two $R$ operators which produces a $N$ operator. In geometrical language, this is reduction of two $R$ punctures to an $\mathbf{N}$ puncture. Likewise, the supersymmetry transformation of an $\mathrm{N}$ operator arises from the geometrical reduction of a surface with an $\mathrm{N}$ puncture and an $\mathrm{R}$ puncture to one with an $\mathrm{R}$ operator. One might expect both of these to have canonical descriptions in terms of the geometry of punctured SRS, and projections of their supermoduli spaces.

\section{Global Structure of SRS with R Punctures}

In Sect. 3 it was found that particularly natural coordinates on an SRS with a pair of $\mathrm{R}$ punctures were $w, \eta$, the torus coordinates, with $\eta=\theta / \sqrt{z}$. In Sect. 4 one encounters similar expressions in $\theta$ (cf. (4.1), (4.4)) which lead us to introduce coordinates with behaviour $\eta \sim \theta / \sqrt{z}$ near the punctures. This in fact simplifies those expressions.

Such coordinates may be introduced globally using an abelian differential $\omega$ with first order poles at punctures; for the moment assume there are poles only 
at the $\mathrm{R}$ punctures, although poles may lie at $\mathrm{N}$ punctures as well. Then define

$$
\eta=\theta \sqrt{\omega_{z}}, \quad w=\int^{z} d z^{\prime} \omega_{z^{\prime}}
$$

There are many such differentials, but a canonical choice is the canonical abelian differential [17], which is uniquely determined (given real numbers, whose sum is zero, for each of the punctures) by the requirement that its periods be pure imaginary. This differential gives the light-cone diagrams [18].

In such coordinates $w, \eta$ the Eqs. (4.1), (4.4) simplify, giving

$$
\begin{array}{ll}
\theta_{a}+\alpha=\eta, & z_{a}+\theta_{a} \alpha=w, \\
\eta_{c}+\gamma=\eta, & w_{c}+\eta_{c} \gamma=w,
\end{array}
$$

etc. One also finds that the zeros of the abelian differential, which correspon to interaction points in the language of string scattering, must correspond to branch points of $\eta .^{9}$

This relation to light-cone diagrams (or more generally, diagrams where the surface is built from flat cylinders, i.e. string propagators) enhances the geometrical picture of the continuation of the results of Sect. 4 to higher genus. It should also be useful in extending the unitarity arguments of [3] to the case of external Ramond states, particularly by supplying a geometrically straightforward description of the integral over supermoduli. Finally, it should be useful in string field theory constructions.

\section{Conclusion}

In this paper a fairly explicit discussion of the construction of punctured super Riemann surfaces has been presented, with a key role played by the definition of standard punctures on SRS. Specific examples of super-spheres with various puncture configurations have been treated in some detail.

In particular, the basic formalism underlying the construction of Ramondpunctured super Riemann surfaces and their supermoduli spaces has been presented. Such constructions, combined with the operator formalism, may be very useful in attacking the general problem of describing fermionic or $(R, R)$ sector backgrounds in the superstring. Specifically, a systematic description of the deformation of superconformal field theories by Neveu-Schwarz backgrounds was given in [19], but it was noted that analysis was not directly applicable to the issue of Ramond backgrounds due to the lack of an explicit understanding of the projection eliminating spin punctures. The formalism indicates how such a projection could be constructed; one can replace a patch containing two $R$ punctures with an unpunctured neighborhood or a standard $\mathrm{N}$ puncture. (In particular, the results of the preceding section may be useful in constructing such a projection and demonstrating its canonical nature.) One may thus attempt to extend that formalism to Ramond sector backgrounds. The results of [19] included an explicit representation for the modification of states due to NS backgrounds. Extension of these formulas to

\footnotetext{
9 One can easily understand this on physical grounds by considering scattering processes involving external or internal Neveu-Schwarz states; for such states the coordinate $\eta$ should be antiperiodic as one travels around the cylinder corresponding to the propagator of that state. This can only happen if $\eta$ is branched at the interaction points
} 
fermionic backgrounds are anticipated for Ramond sector backgrounds. There should be similar expressions shifting Ramond sector states by terms linear in the background, and Neveu-Schwarz sector states by terms quadratic in the background. Likewise it should be possible to derive expressions for the low-order shifts of the super stress tensor as in [19].

A description of $R$ punctures such as that formulated in this paper should also be useful in completing arguments [3] for the unitarity of the super heterotic strings. Application of this formalism should likewise aid in attacking the long-standing problem of deriving the equations of motion for fermionic or $(R, R)$ sector fields in the super and heterotic strings.

Acknowledgements. I wish to thank E. D'Hoker and A. Strominger for useful conservations, and $P$. Nelson for rekindling my interest in issues regarding $R$ punctures and for valuable conversations and comments on an earlier draft.

Note added in proof: A related definition of $\mathbf{R}$ punctures previously appeared in [20].

\section{References}

1. Nelson, P.: Lectures on supermanifolds and strings. In: Particles, Strings, and Supernoavae (Proceedings of 1988 TASI). Jevicki, A., Tan, C.-I. (eds.) Singapore: World Scientific 1989

2. Giddings, S. B.: A brief introduction to super Riemann surface theory. In: Superstrings '88 (Proceedings of 1988 Trieste Spring School). Green, M. et al. (eds.) Singapore: World Scientific 1989

3. Aoki, K., D'Hoker, E., Phong, D. H.: Unitarity of closed superstring perturbation theory. Nucl. Phys. B342, 149 (1990)

4. Vafa, C.: Operator formulation on Riemann surfaces. Phys. Lett. 190B, 47 (1987)

5. Alvarez-Gaumé, L., Gomez, C., Moore, G., Vafa, C.: Strings in the operator formalism. Nucl. Phys. B303, 455 (1988)

6. Alvarez-Gaumé, L., Gomez, C., Nelson, P., Sierra, G., Vafa, C.: Fermionic strings in the operator formalism. Nucl. Phys. B311, 333 (1988)

7. La, H., Nelson, P.: Unambiguous fermionic-string amplitudes. Phys. Rev. Lett. 63, 24(1989)

8. D'Hoker, E., Phong, D. H.: The geometry of string perturbation theory. Rev. Mod. Phys. 60, 917 (1988)

9. Giddings, S. B., Nelson, P., Vafa, C.: unpublished

10. Cohn, J.: Modular geometry of superconformal field theory. Nucl. Phys. B306, 239 (1988)

11. Deligne, P.: unpublished

12. Knizhnik, V.: Covariant superstring amplitudes from the sum over fermionic surfaces. Phys. Lett. 178B, 21 (1986)

13. Friedan, D., Martinec, E., Shenker, S.: Conformal invariance, supersymmetry, and string theory. Nucl. Phys. B271, 93 (1986)

14. Verlinde, E., Verlinde, H.: Lectures on string perturbation theory. In: Superstrings '88 (Proceedings of 1988 Trieste Spring School). Green, M. et al. (eds.) Singapore: World Scientific 1989

15. Giddings, S. B., Martinec, E.: Conformal geometry and string field theory. Nucl. Phys. B278 91 (1986)

16. Friedan, D.: Notes on string theory and two dimensional conformal field theory. Unified String Theories. Green, M., Gross, D. (eds.) Singapore: World Scientific 1986

17. Giddings, S. B., Wolpert, S. A.: A triangulation of moduli space from light-cone string theory. Commun. Math. Phys. 109, 177 (1987)

18. Mandelstam, S.: Dual resonance models. Phys. Rep. 13, 259 (1974)

19. Campbell, M., Nelson, P., Wong, E.: Stress tensor perturbations in conformal field theory. U. of Penn. preprint, Mod. Phys. Lett. A (in press)

20. Baranov, M. A., Schwarz, A. S.: On the multiloop contribution to the string theory. Int. J. Mod. Phys. A2, 1773 (1987) 